Удк 621.3317 .08

\title{
Г.Г. Порубов
}

\section{Алгоритм устранения неоднозначности и вычисления пеленга в многобазовых фазовых пеленгаторах}

\begin{abstract}
Предлагается алгоритм устранения неоднозначности и вычисления пеленга в многобазовых фазовых пеленгаторах по результатам измерения разности фаз. Алгоритм применим для пеленгаторов, оснащённых линейной антенной решёткой с любым числом фазометрических баз.

Ключевые слова: пеленгатор, разность фаз, разрешение неоднозначности, пеленг.

doi: $10.21293 / 1818-0442-2018-21-1-22-27$
\end{abstract}

В многобазовых фазовых пеленгаторах для устранения неоднозначности и вычисления пеленга необходимой и достаточной информацией является набор разностей фаз, полученных при измерении. Функция устранения неоднозначности и вычисления пеленга возлагается на вычислитель пеленга, работающий по принятому алгоритму.

Известен алгоритм устранения неоднозначности, работающий по принципу максимума правдоподобия [1-4].

В статье рассматривается алгоритм, в основу которого положен метод последовательного раскрытия неоднозначности [5]. По результатам измерения разностей фаз на максимальной базе вычисляются ожидаемые значения измеренных разностей фаз на дополнительных базах с последующим сравнением с результатами измерения разностей фаз на этих базах. Условно назовём алгоритм, как алгоритм измеренных разностей фаз.

Максимальную базу пеленгатора обозначим символом $e_{x 1}$, а дополнительные базы - символом $e_{x i}$. Предположим, что ошибки фазовых измерений отсутствуют, а измерения разностей фаз на всех базах производятся неоднозначно.

Полная разность фаз сигналов на базе $e_{x 1}$

$$
\Phi_{1}=2 \pi e_{x 1} \sin \alpha=\varphi_{1}+2 \pi k_{1},
$$

где $e_{x 1}=l_{1} / \lambda-$ база в $\lambda$ (далее - база в целых числах); $\alpha-$ угол прихода плоской волны, отсчитанный от нормали к антенной решетке; $\varphi_{1}$ - измеренная разность фаз; $k_{1}= \pm 0,1,2, \ldots-$ число полных периодов разности фаз $\Phi_{1}$ на базе $e_{x 1}$, утраченных при измерениях в силу периодичности сигналов.

Полную разность фаз на базе $e_{x i}$ получим при умножении (1) на отношение $e_{x i} / e_{x 1}$

$$
\Phi_{i}=2 \pi e_{x i} \sin \alpha=\left(\varphi_{1}+2 \pi k_{1}\right) e_{x i} / e_{x 1},
$$

где $e_{x i}=l_{i} / \lambda$ - базы в $\lambda$ (далее - базы в целых числах).

Из формулы (2) следует выражение для расчёта ожидаемой величины измеренной разности фаз на базе $e_{x i}$

$$
\varphi_{\rho i}=\left(\varphi_{1}+2 \pi k_{1}\right) e_{x i} / e_{x 1}+2 \pi k_{i}
$$

где $k_{i}= \pm 0,1,2, \ldots-$ число полных периодов разности фаз на базе $e_{x i}$, утрачиваемых при вычислениях.

Из формулы (3) следует, что значения ожидаемых величин измеренных разностей фаз $\varphi_{\rho i}$ для дополнительных баз могут быть получены по результатам измерения разности фаз на базе $e_{x 1}$ для любого значения $k_{1}$.

Устранение неоднозначности измерений разности фаз на базе $e_{x 1}$ заключается в отыскании единственного значения $k_{1}$ при совместной обработке результатов измерения разностей фаз на всех базах.

Сравнение ожидаемых величин разностей фаз $\varphi_{\rho i}$ (3) с измеренными значениями разностей фаз $\varphi_{i}$ одновременно по всем дополнительным базам $e_{x i}$ позволит из полного набора величин $k_{1}$ найти искомое значение, определяющее число полных периодов разности фаз, утраченных при измерении разности фаз $\varphi_{1}$ на максимальной базе $e_{x 1}$.

Критерием принятия решения об устранении неоднозначности является равенство ожидаемых величин разностей фаз $\varphi_{\rho i}$ (3) и измеренных значений разностей фаз $\varphi_{i}$ одновременно по всем дополнительным базам $e_{x i}$.

Реально измерения разностей фаз на любой из баз выполняются с ошибками. При определении искомого числа потерянных периодов $k_{1}$ совпадение ожидаемых значений разностей фаз $\varphi_{\rho i}$ (3) и измеренных разностей фаз на дополнительных базах оценивается в некотором фазовом интервале. Совпадение с максимальной вероятностью будет для истинного значения $k_{1}$. Данное предположение положено в основу алгоритма устранения неоднозначности и вычисления пеленга.

Исходные данные для работы алгоритма: $\mathbf{e}_{x}-$ вектор взаимно простых чисел, определяющих выбранную структуру антенной решетки; $v_{\text {раб }}=\sin \alpha_{\text {раб }}-$ размер рабочего сектора; где $\alpha_{\text {раб }}$ - половина сектора пеленгации, отсчитанного от нормали к антен- 
ной решетке; $z_{\varphi i}= \pm 0,5 \Delta_{i}-$ величины разрешенных зон по фазе для дополнительных баз $e_{x i}$,

$$
z_{\varphi i}= \pm 0,5 \Delta_{i}
$$

где $\Delta_{i}-$ величина отклонения измеренной разности фаз на базе $e_{x i}$ с направлений, отличных от истинного пеленга, для которых на базе $e_{x 1}$ разность фаз изменяется на $2 \pi k_{1}$ радиан.

Величины $\Delta_{i}$ задавались при расчёте антенных структур многобазовых фазовых пеленгаторов. Для конкретной структуры антенной решетки величины $\Delta_{i}$ для дополнительных баз приведены в таблице [6, с. 31].

Величина $k_{1}(1)$ определяет число полных периодов разности фаз утрачиваемых при измерении разности фаз на максимальной базе при изменении азимута в пределах рабочего сектора пеленгатора. Для базы $e_{x 1}$ при размере рабочего сектора $v_{\text {раб }}$ максимально возможные значения $k_{1}$ оцениваются по формуле

$$
k_{1}= \pm\left\langle e_{x 1} v_{\text {раб }}\right\rangle,
$$

где $\langle\cdot\rangle$ - операция округления до ближайшего целого.

Измеренные разности фаз представим в пределах $-\pi \leq \varphi_{i} \leq \pi$ и будем измерять в рад/2 $\pi$, так что $-0,5 \leq \varphi_{i} \leq 0,5$. Тогда результат измерения разности фаз на любой из баз с учётом длины волны пеленгуемого сигнала можно вычислить по формуле

$$
\varphi_{i}=\frac{l_{i}}{\lambda} \sin \alpha-\left\langle\frac{l_{i}}{\lambda} \sin \alpha\right\rangle,
$$

где $l_{i} / \lambda$ - база в длинах волн пеленгуемого сигнала; $\alpha$ - угол прихода плоской волны, отсчитанный относительно нормали к антенной решетке; $\langle\cdot\rangle$ - операция округления до ближайшего целого.

Величина $\left\langle\frac{l_{i}}{\lambda} \sin \alpha\right\rangle$ в (6) определяет искомое число потерянных периодов $k_{i}$ при измерении разности фаз на базе $e_{x i}$.

\section{Алгоритм}

Итак, имеется вектор измеренных разностей фаз $\varphi_{i}(6)$.

По разности фаз $\varphi_{1}$, измеренной на максимальной базе, подставляя последовательно значения чисел $k_{1}(5)$, определяющих число полных периодов разности фаз, утрачиваемых при измерении разности фаз на базе $e_{x 1}$, находятся величины ожидаемых значений измеренных разностей фаз на дополнительных базах ехі по формуле

$$
\varphi_{\rho i}=\left(\varphi_{1}+k_{1}\right) e_{x i} / e_{x 1}-\left\langle\left(\varphi_{1}+k_{1}\right) e_{x i} / e_{x 1}\right\rangle,
$$

где $k_{1}$ - число полных периодов разности фаз, утрачиваемых при измерениях на максимальной базе; $\langle\cdot\rangle$ - операция округления до ближайшего целого.

Определяются разности

$$
\psi_{i}=\left(\varphi_{i}-\varphi_{\rho i}\right)-\left\langle\left(\varphi_{i}-\varphi_{\rho i}\right)\right\rangle,
$$

где $\varphi_{\rho i}$ - результат вычисления ожидаемой величины измеренной разности фаз для базы $e_{x i}(7) ; \varphi_{i}-$ результат измерения разности фаз на базе $e_{x i}(6)$; $i=2,3, \ldots, n ;\langle\cdot\rangle-$ операция округления до ближайшего целого.

Условие правильного устранения неоднозначности запишется в виде

$$
\left|\psi_{i}\right| \leq z_{\varphi i}
$$

где $z_{\varphi i}$ - величина разрешенной зоны по фазе (4); $i=2,3, \ldots, n$.

При невыполнении условия (9) хотя бы по одной из дополнительных баз неоднозначность не разрешена. Далее в выражение (7) подставляется очередное значение числа $k_{1}$ с последующей оценкой условия правильного устранения неоднозначности (9).

При выполнении условия (9) по всем дополнительным базам принимается решение об устранении неоднозначности и вычисляется параметр

$$
\sin \alpha_{1}=\frac{\varphi_{1}+k_{1}}{e_{x 1}},
$$

где $\varphi_{1}-$ измерения разность фаз на базе $e_{x 1} ; k_{1}-$ значение числа, определяющее число полных периодов разности фаз, утраченных при измерении разности фаз на базе $e_{x 1}$, при котором выполняется условие (9).

Измерение разности фаз производится для баз пеленгатора, выраженных в длинах волн пеленгуемого сигнала (6), а устранение неоднозначности и вычисление параметра $\sin \alpha_{1}$ (10) выполняются для баз, которые представлены в целых числах. Для приведения найденного значения $\sin \alpha_{1}$ к величине, соответствующей данному азимуту с учётом максимальной базы, выраженной в длине волны пеленгуемого сигнала, необходимо значение $\sin \alpha_{1}(10)$ умножить на частотный коэффициент

$$
K_{f}=f_{0} / f_{i},
$$

где $f_{i}$ - частота пеленгуемого источника излучения; $f_{0}$ - частота, соответствующая длине волны, величина которой определяется по формуле $\lambda_{0}=l_{i} / e_{x i}$, где $l_{i}$, см - расстояние между антенными излучателями базы; $e_{x i}-$ база, выраженная в целых числах.

Значение частоты $f_{0}$ определяется по формуле

$$
f_{0}=c / \lambda_{0},
$$

где $c=3 \cdot 10^{10} \mathrm{~cm} \cdot \mathrm{c}^{-1}-$ скорость света в вакууме.

При выполнении неравенства $\left|K_{f} \sin \alpha_{1}\right| \leq v_{\text {раб }}$ измеренный пеленг вычисляется по формуле

$$
\alpha_{\text {изм }}=\arcsin \left(K_{f} \sin \alpha_{1}\right),
$$

где $K_{f}$ - частотный коэффициент (11). 
Оценка искомого пеленга может быть выполнена, при целесообразности, по результатам измерения разности фаз на всех или части баз пеленгатора, т.е. по суммарной базе, при условии восстановления полной разности фаз на каждой из баз пеленгатора.

Число полных периодов разности фаз, утраченных при измерении разности фаз на дополнительных базах, определяется по формуле

$$
k_{i}=\left\langle\left(\varphi_{1}+k_{1}\right) e_{x i} / e_{x 1}\right\rangle+\left\langle\left(\varphi_{\rho i}-\varphi_{i}\right)\right\rangle,
$$

где $i=2,3, \ldots, n$.

Полная разность фаз на $i$-й базе пеленгатора

$$
\Phi_{i}=e_{x i} \sin \alpha=\varphi_{i}+k_{i} .
$$

Сумма полных разностей фаз ряда баз равна

$$
\sum_{i=1}^{n} \Phi_{i}=\sum_{i=1}^{n} e_{x i} \sin \alpha=\sum_{i=1}^{n} \varphi_{i}+\sum_{i=1}^{n} k_{i} .
$$

Тогда вычисление искомого параметра при оценке по суммарной базе выполняется по формуле

$$
\sin \alpha_{2}=\frac{\sum_{i=1}^{n} \varphi_{i}+\sum_{i=1}^{n} k_{i}}{\sum_{i=1}^{n} e_{x i}},
$$

где $\varphi_{i}-$ результат измерения разности фаз на $i$-й базе; $k_{1}$ - значение числа, при котором выполняется условие (9); $k_{i}$ - число полных периодов разности фаз, утраченных при измерении разности фаз на дополнительных базах (13); $i=1,2,3, \ldots, n$.

При выполнении неравенства $\left|K_{f} \sin \alpha_{2}\right| \leq v_{\text {раб }}$ измеренный пеленг вычисляется по (12).

\section{Функциональное резервирование}

Фазовый пеленгатор при отказе одного из измерителей переходит в нерабочее состояние.

Способ повышения надежности, состоящий в дублировании пеленгатора, т.е. включение в состав аппаратуры двух комплектов пеленгаторов, не оптимален. Во-первых, отказ одного из измерителей в каждом из пеленгаторов ведёт к отказу обеих пеленгаторов. Во-вторых, для реализации двух $n$-базовых пеленгаторов необходимо установить две антенные решетки с общим числом антенных излучателей $2 n+2$, размещение которых может оказаться проблематичным.

Предлагается другой способ повышения надёжности пеленгатора, состоящий в объединении двух $n$-базовых пеленгаторов через максимальную базу в пеленгатор с числом баз $2 n-m$. Кроме уменьшения габаритов антенной решетки, числа антенных излучателей и числа приёмных каналов, такая схема пеленгатора допускает отказ ряда измерителей пеленгатора без потери работоспособности.

При отказе измерителя, без которого работа пеленгатора невозможна, необходимо восстановить виртуальное значение измеренной разности фаз, аналогичное реальному значению измеренной разности фаз отказавшего измерителя. Для восстанов- ления виртуальной величины измеренной разности фаз используются результаты измерения разности фаз других измерителей. Способ восстановления виртуальной разности фаз определяется способом восстановления виртуальной базы отказавшего измерителя.

Рассмотрим подход к выбору антенной структуры пеленгатора. Выбор величины максимальной базы определяется требованием обеспечения заданной точности пеленгования. Дополнительные базы антенной структуры каждого из $n$-базовых пеленгаторов в общем случае не равны и выбираются с учетом возможности размещения антенных излучателей в антенной структуре с максимальным приближением к оптимальным структурам [7]. Кроме этого, при выборе величин дополнительных баз антенных структур следует учитывать возможность получения виртуальных баз величиной, равной величинам баз отказавших измерителей. Виртуальные базы находятся по формуле

$$
e_{v i}=\sum_{l=1}^{n} e_{x(i \pm l)},
$$

где $e_{v i}$ - виртуальная база, равная по величине базе $e_{x i}$, отказавшего измерителя; $e_{x(i \pm l)}$ - дополнительные базы, обеспечивающие получение необходимой величины виртуальной базы.

После определения виртуальной базы (14) необходимо вычислить виртуальное значение измеренной разности фаз по формуле

$$
\varphi_{v i}=\sum_{l=1}^{n} \Phi_{i \pm l}-\left\langle\sum_{l=1}^{n} \Phi_{i \pm l}\right\rangle,
$$

где $\Phi_{i \pm l}=\varphi_{i \pm l}+k_{i \pm l}-$ полная разность фаз на базе $e_{x(i \pm l)}$, где $\varphi_{i \pm l}-$ результат измерения разности фаз на базе $e_{x(i \pm l)} ; k_{i \pm l}$ - число полных периодов разности фаз, утраченных при измерении на базе $e_{x(i \pm l)}(13) ;\langle\cdot\rangle-$ операция округления до ближайшего целого.

Идентичность виртуальной и реальной баз оценивается по равенству математических ожиданий измеренных разностей фаз виртуальной и реальной баз в пеленгах неоднозначности максимальной базы. Под пеленгами неоднозначности максимальной базы следует понимать направления, отличные от истинного пеленга, для которых на максимальной базе разность фаз изменяется на $2 \pi k_{1}$ радиан.

Величины математических ожиданий измеренной разности фаз реальной базы $e_{x i}$ находятся по формуле [7]

$$
m_{i j}=\left(\frac{e_{x i}}{e_{x 1}}\right) j-\left\langle\left(\frac{e_{x i}}{e_{x 1}}\right) j\right\rangle,
$$

где $j=0,1,2, \ldots,(h-1)$ - номер пеленга неоднозначности максимальной базы $e_{x 1}=h ;\langle\cdot\rangle-$ операция округления до ближайшего целого. 
Величины математических ожиданий измеренной разности фаз виртуальной базы (14) находятся по формуле

$$
m_{v i j}=\sum_{l=1}^{n} m_{(i \pm l) j}-\left\langle\sum_{l=1}^{n} m_{(i \pm l) j}\right\rangle,
$$

где $m_{(i \pm l) j}$ - математические ожидания измеренной разности фаз реальных баз (16), выбранных для формирования виртуальной базы.

Рассмотрим режим функционального резервирования на примере шестибазового фазового пеленгатора с вектором баз

$$
\vec{e}_{x}=\left(e_{x 1}, e_{x 2}, e_{x 3}, e_{x 4}, e_{x 5}, e_{x 6}\right) .
$$

Пеленгатор образован из двух схем четырёхбазовых пеленгаторов с векторами баз [6]

$$
\begin{aligned}
& \vec{e}_{x}=\left(e_{x 1}, e_{x 2}, e_{x 3}, e_{x 4}\right)=(12,6,10,7), \\
& \vec{e}_{x}=\left(e_{x 1}, e_{x 2}, e_{x 5}, e_{x 6}\right)=(12,6,4,3) .
\end{aligned}
$$

Схема антенной решетки шестибазового фазового пеленгатора приведена на рис. 1.

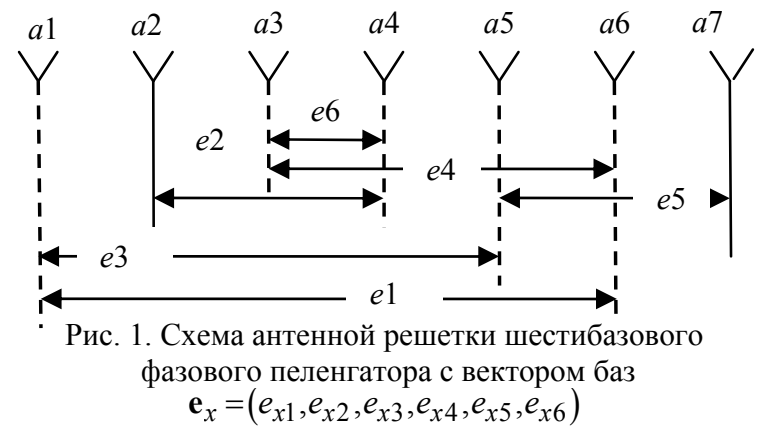

Для пар баз антенной структуры (см. рис. 1) величины коэффициентов корреляции ошибок измерения разности фаз равны:

$$
\begin{gathered}
e_{x 1}-e_{x 3} \quad r=0,5 ; e_{x 1}-e_{x 4} \quad r=0,5 ; e_{x 2}-e_{x 6} \quad r=0,5 ; \\
e_{x 3}-e_{x 5} \quad r=-0,5 \text { и } e_{4}-e_{x 6} \quad r=0,5 .
\end{gathered}
$$

Для других пар баз в любом сочетании коэффициенты корреляции ошибок измерения разности фаз равны нулю.

В пеленгаторе (см. рис. 1) с вектором баз (17) допускается отказ одновременно нескольких пар измерителей с базами $e_{x 3}$ и $e_{x 4}, e_{x 5}$ и $e_{x 6}, e_{x 3}$ и $e_{x 6}$ или $e_{x 4}$ и $e_{x 5}$ без потери работоспособности и без формирования виртуальных баз.

При отказе ряда измерителей величины виртуальных баз находятся по формулам: при отказе измерителя $e x 2$

$$
e_{v 2}=e_{x 3}-e_{x 5}=6,
$$

при отказе измерителей $e_{x 3}$ и $e_{x 5}$

$$
e_{v 3}=e_{x 4}+e_{x 6}=10 \text {, или } e_{v 5}=e_{x 4}-e_{x 6}=4,
$$

при отказе измерителей $e_{x 2}$ и $e_{x 3}$

$$
e_{v 23}=e_{x 4}+e_{x 6}-e_{x 5}=6,
$$

при отказе измерителей $e_{x 2}$ и $e_{x 5}$

$$
e_{v 25}=e_{x 3}-e_{x 4}+e_{x 6}=6,
$$

при отказе измерителей $e_{x 2}, e_{x 3}$ и $e_{x 4}$

$$
e_{v 26}=2 e_{x 6}=6 .
$$

Ошибки измерения разности фаз на виртуальных базах (14) зависят от числа реальных баз, которые выбраны для формирования виртуальной базы, и от величины коэффициента корреляции ошибок измерения разности фаз на этих базах.

Базовые ошибки измерения разности фаз на реальных базах - случайные величины. Тогда среднеквадратические ошибки суммы (или разности) произвольного числа случайных величин вычисляется по формуле [8]

$$
\sigma_{v i}=\sqrt{\sum_{i=1}^{n} \sigma_{\varphi i}^{2} \pm 2 \sum_{i<j} r_{i j} \sigma_{\varphi i} \sigma_{\varphi j}},
$$

где $\sigma_{\varphi i}$ - базовые фазовые ошибки; $r_{i j}$ - коэффициент корреляции ошибок измерения разности фаз.

Знак «+» под корнем в (18) соответствует определению виртуальной базы как сумма реальных баз, а знак «минус» - определению виртуальной базы как разность реальных баз. Второй знак суммы определяет наличие не менее двух пар баз, коэффициент корреляции ошибок измерения разности фаз которых отличен от нуля.

Величины фазовых ошибок виртуальных баз, вычисленных по формуле (18), приведены в табл. 1.

Правильное устранение неоднозначности определяется условием (9) и зависит от величины фазовых ошибок разностей $\psi_{i}(8)$. Рассмотрим случай приёма сигнала с направления $\alpha=0$. Измеренные разности фаз определяются ошибками приёмных каналов, это случайные величины с равными дисперсиями $\sigma_{\varphi i}^{2}$.

Если при вычислении разности фаз $\psi_{i}(8)$ применена измеренная разность фаз $\varphi_{i}$ реальной базы $e_{x i}$, то величина фазовых ошибок разности фаз $\psi_{i}$ вычисляется по формуле

$$
\begin{gathered}
\sigma_{\psi i}=\sqrt{\sigma_{\varphi i}^{2}+\sigma_{\varphi i}^{2}\left(\frac{e_{x i}}{e_{x 1}}\right)^{2}-2 r \sigma_{\varphi i} \sigma_{\varphi i} \frac{e_{x i}}{e_{x 1}}}= \\
=\sqrt{\frac{\sigma_{\varphi i}^{2} e_{x 1}^{2}+\sigma_{\varphi i}^{2} e_{x i}^{2}-2 r \sigma_{\varphi i} \sigma_{\varphi i} e_{x 1} e_{x i}}{e_{x 1}^{2}}} .
\end{gathered}
$$

Если при вычислении разности фаз $\psi_{i}(8)$ применена измеренная разность фаз виртуальной базы $\varphi_{v i}(15)$, то величина фазовых ошибок разности фаз $\psi_{i}$ вычисляется по формуле

$$
\begin{gathered}
\sigma_{\psi i}=\sqrt{\sigma_{v i}^{2}+\sigma_{\varphi i}^{2}\left(\frac{e_{v i}}{e_{x 1}}\right)^{2}-2 \eta_{1 i} \sigma_{v i} \sigma_{\varphi i} \frac{e_{v i}}{e_{x 1}}}= \\
=\sqrt{\frac{\sigma_{v i}^{2} e_{x 1}^{2}+\sigma_{\varphi i}^{2} e_{v i}^{2}-2 r_{1 i} \sigma_{v i} \sigma_{\varphi i} e_{x 1} e_{v i}}{e_{x 1}^{2}}},
\end{gathered}
$$

где $\sigma_{\varphi i}-$ базовые фазовые ошибки реальной базы; $\sigma_{v i}-$ фазовые ошибки виртуальной базы (18); $\eta_{i}-$ коэффициент корреляции ошибок измерения разности фаз на базах $e_{x 1}$ и $e_{v i}$. 
Значение коэффициентов корреляции $\eta_{1 i}$ определены методом математического моделирования. Полученные результаты приведены в табл. 1 .

Выполнена сравнительная оценка вероятностей правильного устранения неоднозначности пеленгаторов с различным вектором относительных баз по методике, предложенной в [7]. Вероятность правильного устранения неоднозначности вычислялась по формуле

$$
P_{0 i}=\int_{-z_{\varphi i}}^{z_{\varphi i}} \ldots \int_{-z_{\varphi n}}^{z_{\varphi n}} w_{n-1}\left(y_{2}, \ldots, y_{n}\right) d y_{2} \ldots d y_{n},
$$

где $n$ - число баз пеленгатора; $z_{\varphi i}$ - разрешенная зона по фазе базы $e_{x i}(4) ; w_{n-1}\left(y_{2}, \ldots, y_{n}\right)$ - плотность распределения вероятностей случайной величины $\psi_{i}(8)$.

Т а бли и а 1

Фазовые ошибки виртуальных баз и коэффициенты корреляции

\begin{tabular}{|c|c|c|}
\hline \multirow{2}{*}{ Виртуальные базы } & $\begin{array}{c}\text { Фазовые } \\
\text { ошибки }\end{array}$ & $\begin{array}{c}\text { Коэффициент } \\
\text { корреляции }\end{array}$ \\
\cline { 2 - 3 } & $\sigma_{v i}$, град & $\eta_{1 i}$ \\
\hline$e_{v 2}=e_{x 3}-e_{x 5}$ & $\sqrt{3} \sigma_{\varphi l}$ & 0,27 \\
\hline$e_{v 3}=e_{x 4}+e_{x 6}$ & $\sqrt{3} \sigma_{\varphi l}$ & 0,27 \\
\hline$e_{v 5}=e_{x 4}-e_{x 6}$ & $\sigma_{\varphi l}$ & 0,5 \\
\hline$e_{v 23}=e_{x 4}+e_{x 6}-e_{x 5}$ & $2 \sigma_{\varphi 1}$ & 0,2 \\
\hline$e_{v 25}=e_{x 3}-e_{x 4}+e_{x 6}$ & $2 \sigma_{\varphi l}$ & 0 \\
\hline$e_{v 26}=2 e_{x 6}$ & $\sqrt{2} \sigma_{\varphi 1}$ & 0 \\
\hline
\end{tabular}

Кроме этого, проведена оценка вероятностей правильного устранения неоднозначности этих же пеленгаторов методом математического моделирования. Результаты вычислений приведены в табл. 2.

Вероятности правильного устранения неоднозначности $P_{01}$ и $P_{03}$ вычислены по (21) при задании фазовых ошибок, распределённых по нормальному закону, величинами $\sigma_{\varphi i}=36^{\circ}$ и $\sigma_{\varphi i}=27^{\circ}$ соответственно. Вероятности правильного устранения неоднозначности $P_{02}$ и $P_{04}$ получены по результатам математического моделирования при величинах фазовых ошибок $\sigma_{\varphi i}=36^{\circ}$ и $\sigma_{\varphi i}=27^{\circ}$ соответственно.

Таблица 2

Результаты оценки вероятности правильного устранения неоднозначности

\begin{tabular}{|c|l|c|c|c|}
\hline \multirow{2}{*}{$\begin{array}{c}\text { Вектор } \\
\text { относительных }\end{array}$} & \multicolumn{3}{|c|}{$\begin{array}{l}\text { Вероятности правильного } \\
\text { баз }\end{array}$} & \multicolumn{2}{|c|}{$P_{01}$} & $P_{02}$ & \multicolumn{2}{|c|}{$P_{03}$} & $P_{04}$ \\
\cline { 2 - 5 } & \multicolumn{2}{|c|}{$\sigma_{\varphi i}=36^{\circ}$} & \multicolumn{2}{|c|}{$\sigma_{\varphi i}=27^{\circ}$} \\
\hline$e_{x 1}, e_{x 2}, e_{x 3}, e_{x 4}$ & 0,9 & 0,902 & 0,98 & 0,98 \\
\hline$e_{x 1}, e_{x 2}, e_{x 5}, e_{x 6}$ & 0,85 & 0,853 & 0,961 & 0,962 \\
\hline$e_{x 1}, e_{x 2}, e_{v 3}, e_{x 4}$ & 0,785 & 0,844 & 0,929 & 0,949 \\
\hline$e_{x 1}, e_{v 2}, e_{x 3}, e_{x 4}$ & 0,651 & 0,66 & 0,805 & 0,808 \\
\hline$e_{x 1}, e_{x 2}, e_{v 5}, e_{x 4}$ & 0,914 & 0,921 & 0,984 & 0,985 \\
\hline$e_{x 1}, e_{v 23}, e_{x 5}, e_{x 6}$ & 0,697 & 0,731 & 0,878 & 0,89 \\
\hline$e_{x 1}, e_{v 25}, e_{x 3}, e_{x 4}$ & 0,716 & 0,874 & 0,879 & 0,966 \\
\hline$e_{x 1}, e_{v 26}, e_{x 5}, e_{x 6}$ & 0,789 & 0,689 & 0,938 & 0,862 \\
\hline$e_{x 1}, e_{x 2}, e_{x 3}, e_{x 4}, e_{x 5}, e_{x 6}$ & 0,786 & 0,803 & 0,945 & 0,947 \\
\hline
\end{tabular}

Оценки вероятностных характеристик (см. табл. 2) выполнены при ошибках измерения разно- сти фаз $\sigma_{i \rho}=36^{\circ}$, величина которых выбрана условно, что позволяет сравнить вероятностные характеристики различных антенных структур, приведенных в ряде работ, при равных условиях.

Однако запрета на уменьшение величины фазовых ошибок нет. Последнее позволит при уменьшении фазовых ошибок получить вероятностные характеристики различных антенных структур желаемого уровня.

\section{Выводы}

Алгоритм выполняет устранение неоднозначности и вычисление пеленга по результатам измерения разности фаз в фазовом пеленгаторе с любым числом баз и любой схемой антенной решетки.

При увеличении числа баз антенной решетки алгоритм повышает надёжность пеленгатора методом функционального резервирования. Последнее позволит увеличить время безотказной работы устройства.

Алгоритм может быть применён не только в многобазовых фазовых пеленгаторах, но и в других видах многошкальных фазовых измерителей.

\section{Лuтература}

1. Белов В.И. Алгоритмы устранения неоднозначности в фазовой многоканальной измерительной системе // Радиотехника и электроника. - 1976. - Т. 21, № 8. C. 1657.

2. Белов В.И. Квазиоптимальный алгоритм устранения неоднозначности в многошкальной фазовой измерительной системе // Радиотехника и электроника. - 1990. T. 35, № 8. - С. 1642.

3. Денисов В.П. Максимально правдоподобное разрешение неоднозначности многошкальных фазовых измерений // Известия вузов. Радиоэлектроника. - 1977. T. 20, № 7. - С. 63.

4. Неплохов И.Г. Устройство разрешения многозначности фазовых измерений: А.с. 993146 СССР // Б.И. 1983. - № 4. - С. 227.

5. Денисов В.П. Фазовые радиопеленгаторы / В.П. Денисов, Д.В. Дубинин. - Томск: Изд-во ТУСУР, 2002. $-251 \mathrm{c}$.

6. Порубов Г.Г. Методика расчёта антенных структур многобазовых фазовых пеленгаторов / Г.Г. Порубов, В.П. Денисов // Доклады ТУСУР. - 2015. - № 3 (37). C. $25-32$.

7. Порубов Г.Г. Методика выбора оптимальных структур антенных решеток фазовых пеленгаторов и оценка вероятностных характеристик // Доклады ТУСУР. - 2017. - T. 20, № 1. - С. 5-9.

8. Левин Б.Р. Теоретические основы статистической радиотехники: в 3 кн. - Кн. 1. - М.: Сов. радио, 1969. C. $117,141$.

\section{Порубов Геннадий Гаврилович}

Инженер, ОАО «НИИАП»

Дзержинского пр-т, 87, Новосибирск, Россия, 630051

Тел.: 8 (383) 279-52-28

Эл. почта: porub27@mail.ru 
Porubov G.G.

Algorithm of eliminating ambiguity and calculating

bearing for multibase phase direction finders

The algorithm of eliminating ambiguity and calculating bearing in multibase phase finders according to the results of phase measurements is suggested. The algorithm can be used for direction finders equipped with a linear antenna array having any number of phase measuring bases.

Keywords: direction finder, phase difference, ambiguity resolution, bearing.

doi: $10.21293 / 1818-0442-2018-21-1-22-27$

\section{References}

1. Belov V.I. Algorithms for eliminating ambiguity in a phase multichannel measuring system. Radio engineering and electronics, 1976, vol. 21, no. 8, p. 1657. (In Russ.)

2. Belov V.I. Quasi-optimal algorithm for eliminating ambiguity in a multi-scale phase measuring system. Radio engineering and electronics, 1990, vol. 35, no. 8, p. 1642. (In Russ.)

3. Denisov V.P. Maximum plausible resolution of the ambiguity of multi-scale phase measurements. Izvestiya high schools. Radioelectronics, 1977, vol. 20, no. 7, p. 63. (In Russ.)
4. Neplokhov I.G. The device for resolution of multivaluedness of phase measurements. Patent RF, no. 993146, 1983.

5. Denisov V.P., Dubinin D.V. Phase direction finders. Tomsk,Publishing House TUSUR, 2002. 251 p. (In Russ.)

6. Porubov G.G., Denisov V.P. Method for calculating the antenna structures of multi-phase phase-finders. Doklady TUSUR, 2015, vol. 37, no. 3, pp. 25-32. (In Russ.)

7. Porubov G. G. The method of choosing the optimal structures of antenna arrays of phase-finders and estimation of probabilistic characteristics. Doklady TUSUR, 2017, vol. 20, no. 1, pp. 5-9. (In Russ.)

8. Levin BR Theoretical bases of statistical radio engineering. In 3 Book, Book 1, Moscow, Sov. Radio pabl., 1969, pp. 117, 141. (In Russ.)

\section{Gennadiy G. Porubov}

Engineer, OJSC «NIIAP»

87, Dzerzhinsky Av., Novosibirsk, Russia, 630051

Phone: 8 (383) 279-52-28

Email: porub27@mail.ru 